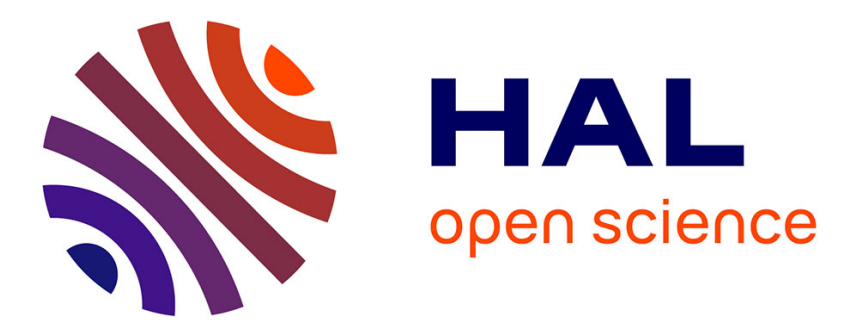

\title{
Thermodynamic study of a lattice of compass needles in dipolar interaction
}

\author{
Enrick Olive, Pierre Molho
}

\section{To cite this version:}

Enrick Olive, Pierre Molho. Thermodynamic study of a lattice of compass needles in dipolar interaction. Physical Review B: Condensed Matter and Materials Physics (1998-2015), 1998, 58 (14), pp.9238 - 9247. 10.1103/PhysRevB.58.9238 . hal-01794557

\section{HAL Id: hal-01794557 \\ https://hal.science/hal-01794557}

Submitted on 17 May 2018

HAL is a multi-disciplinary open access archive for the deposit and dissemination of scientific research documents, whether they are published or not. The documents may come from teaching and research institutions in France or abroad, or from public or private research centers.
L'archive ouverte pluridisciplinaire HAL, est destinée au dépôt et à la diffusion de documents scientifiques de niveau recherche, publiés ou non, émanant des établissements d'enseignement et de recherche français ou étrangers, des laboratoires publics ou privés. 


\title{
Thermodynamic study of a lattice of compass needles in dipolar interaction
}

\author{
Enrick Olive and Pierre Molho \\ Laboratoire Louis Néel, CNRS-UJF, BP 166, 38042 Grenoble cedex, France
}

(Received 13 June 1997; revised manuscript received 13 March 1998)

\begin{abstract}
We present the study of an original system, which is an experimental dipolar model allowing a precise understanding of dipolar effects. This system is a square lattice of $22 \times 22$ magnetized needles (compass needles). Its thermodynamic properties are studied by considering the action of a random external field supposed to mimic the thermal fluctuations. In parallel, a Monte Carlo numerical simulation of the experimental system is performed. Both studies show the existence of a phase transition between an ordered phase and a disordered one. We introduce the notion of "line length" which is the relevant quantity to describe the obtained configurations and to give a precise description of the observed transition. This phase transition is characterized by the emergence of a preferential direction, called the "director," and by an increase of the average line length. The order parameter expressing the symmetry breaking is defined. Our results show an example of a successful mapping of a dynamic dissipative system excited by a random field onto equilibrium statistical mechanics. [S0163-1829(98)05638-0]
\end{abstract}

\section{INTRODUCTION}

In the past, the dipolar interaction was generally not much studied in magnetism. This anisotropic long range interaction was usually neglected in magnetic materials because it is much weaker than the exchange interaction. But since a few years ago, with the growing interest for the mesoscopic physics, the dipolar interaction has become of great importance. The discovery of new properties justifies the interest for such systems. We can cite for example the domain structures in thin films, lattices of magnetic dots and heterogeneous nanocrystallized systems. Furthermore, close collaborations between chemists and physicists give rise to fascinating molecular nanomagnetic systems such as Mn12 and Fe8 clusters. Evidence for quantum tunneling of the magnetization is shown in these systems, ${ }^{1-3}$ and recent relaxation experiments on $\mathrm{Fe} 8$ are interpreted using the dipolar fields. ${ }^{4,5}$ Nevertheless, it is not always straightforward even in these nice systems to have a clear idea of the precise effects of the dipolar interaction since they may be either not measurable at the macroscopic scale, or hidden by various distributions. Thus, the need for a complete and precise understanding of the dipolar effects is necessary and a dipolar model may be of a great interest.

In most of the previous studies, the dipolar interaction was added to the exchange one in order to study its effects on the existence and nature of phase transitions. It has been shown for instance that a dipolar term introduced in the twodimensional (2D) Heisenberg model is able to stabilize a long range magnetic order; see for instance Refs. 6 and 7. For purely 2D dipolar models, theoretical and numerical simulations results show that long range order exists for all Bravais lattices except for the square one. In this case, a "Kosterlitz-Thouless-like transition" is expected, that means with an ordered phase characterized by a power law correlation function; see, e.g., Refs. 7 and 8. However, Prakash and Henley ${ }^{9}$ predict a long range ordered phase resulting from a thermal fluctuation selection effect which breaks the continuous degeneracy of the ground state. This selection induces a discrete fourfold symmetry. This phenomenon is analogous to the "ordering due to disorder", which exists in the geometrically frustrated spins systems. ${ }^{10}$

We present in this paper an original experimental dipolar system made of $22 \times 22$ compass needles on a square lattice. ${ }^{11}$ This system is a dipolar model where the dipolar effects are isolated since they are not mixed with other effects such as for instance the exchange interaction. Therefore many studies are possible with this experimental model system in order to understand precisely the dipolar interaction and its effects. We present here a study of the thermodynamic properties of this experimental dynamic dissipative system by considering the action of a random external field supposed to mimic the thermal fluctuations. As we shall see, the experimental results obtained with such a random field and treated in a thermodynamic approach, are comparable to those obtained from a Monte Carlo numerical simulation where the temperature allows the system to probe the phase space according to the Boltzmann statistics.

The outline of this paper is as follows. In Sec. II we describe the experimental setup which allows the study of these compass needle lattices, and we describe some important first observations of the square lattice behaviors when various fields are applied and in particular random fields. The conclusions of these first observations are compared to the case of the "pure" dipole square lattice. This comparison shows the obvious higher order term effects in our experimental system. In Sec. III we describe the experiments and the Monte Carlo numerical simulation, and the results are presented in Sec. IV. Finally, the interplay between the experiments and the numerical simulation is discussed in Sec. V. A quantitative comparison between them is proposed, leading to the conclusion that the mapping of this dynamical dissipative system excited by a random field onto the equilibrium statistical mechanics is successful.

\section{EXPERIMENTAL SETUP AND LATTICE OF COMPASS NEEDLES BEHAVIORS}

The present study concerns a square lattice of $22 \times 22$ magnetized needles separated by $12 \mathrm{~mm}$. Each needle has a 
rhombic shape with semiaxis lengths $l_{1}=5 \mathrm{~mm}$ and $l_{2}$ $=1.5 \mathrm{~mm}$, and a thickness of $0.2 \mathrm{~mm}$. They are put onto nonmagnetic vertical axes and can rotate in the $(x, y)$ plane. Magnetic fields can be applied in the plane of the lattice by two independent perpendicular current layers. The lattice is shielded from parasitic fields and the earth field by soft magnetic sheet of metal. Residual fields are then compensated by applying a small constant field. The energy injected by the application of various fields is dissipated by weak dry friction due to the contact between compass needles and their vertical axis, and by the viscous damping of the needles in the air. Data acquisition is performed by image processing which allows the measurements of each needle angle (but not the velocities) and to compute all relevant quantities such as potential energy and torques. The accuracy of the measurement depends on the needle velocities and is better than five degrees in this study.

These lattices of magnetized needles are complex systems with many metastable states. They are model systems that can illustrate for example the microscopic magnetic states: ferromagnetic, paramagnetic and domain structures. They also display magnetization reversal processes analogous to what occurs in real magnets, i.e., nucleation and avalanches. However, one should be very cautious with these analogies since the needles interact via dipolar interactions and not via the exchange one.

If a constant field is applied along the square axis directions, strong enough to align all needles, then lowering the field to zero will let that "ferromagnetic" configuration unchanged. This structure is very stable since the demagnetizing field is not strong enough to disrupt it. If a constant field is applied along the $\pi / 4$ direction, strong enough to align all needles, and then switched off, the needles will split up onto the two closest lattice axes, forming lines in these two directions. Now, let us consider the case of a field of random direction and amplitude (chosen between 0 and $H_{\max }$ ) and such that the frequency of this random field, i.e., the rate of change of $H$ in the range $\left[0, H_{\max }\right]$, is close to resonant frequencies of this dynamic system (about $8 \mathrm{~Hz}$ ). If such a field is applied to the lattice, we then observe oscillations of the needles: oscillations weakly disruptive for the lines of needles at low amplitude of the random field, i.e., for small values of $H_{\max }$, and larger oscillations or chaotic rotations with increasing $H_{\max }$, leading to short line lengths with short lifetimes. Thus, for high values of $H_{\max }$, we observe a disordered state which can illustrate the paramagnetic one (see Fig. 1). If the applied random field is then decreased very slowly, on different places of the lattice the needles manage to form antiparallel lines but some places of the lattice show parallel lines of needles. In the same way, if we switch off quickly the random excitation we then obtain a trapped configuration where the needles form lines of different sizes in the four lattice directions (see Fig. 2).

Two main conclusions can be drawn from these first observations. The first one is the obvious existence of four anisotropy directions which correspond to the lattice axes. The second conclusion is the tendency of the system to form lines of needles (along the square lattice axes).

The origin of these observed anisotropy effects can be clarified by comparison with the square lattice of "pure"

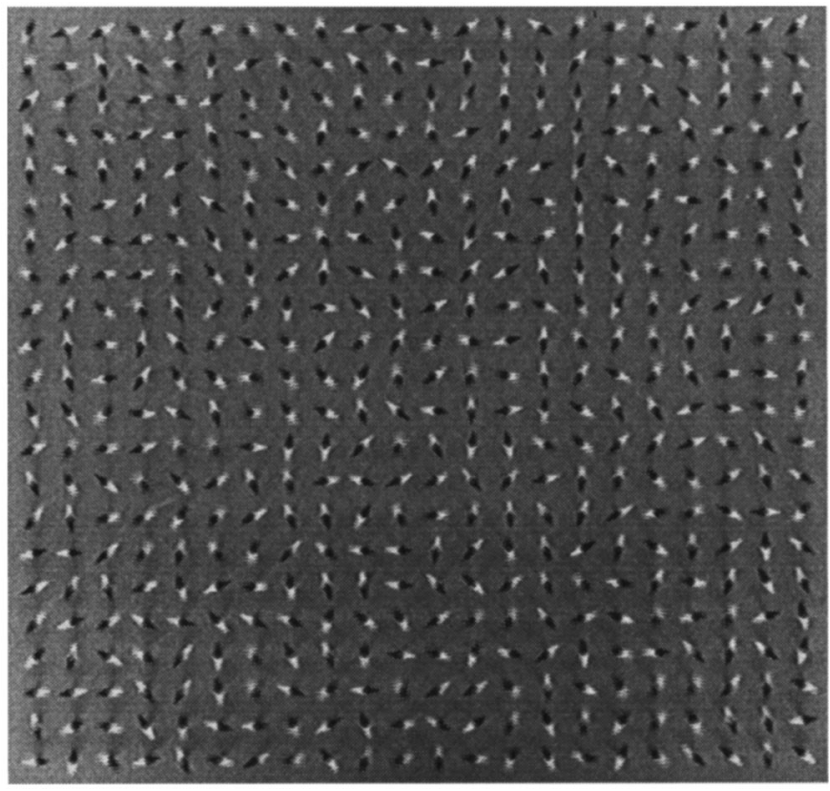

FIG. 1. Experimental snapshot of a disordered configuration obtained while applying a field of random amplitude and direction. The amplitude is chosen between 0 and $H_{\max }$. The frequency of this random field, i.e., the rate of change of $H$ in the range $\left[0, H_{\max }\right]$, is close to resonant frequencies of this dynamic system, i.e., about 8 $\mathrm{Hz}$.

dipoles. The dipolar interaction is described by the Hamiltonian:

$$
H_{\mathrm{dip}}=\frac{1}{2} \sum_{i} \sum_{j, j>i} \frac{1}{\mathbf{r}_{i j}^{3}}\left[\mu_{i} \mu_{j}-\frac{3}{\mathbf{r}_{i j}^{2}}\left(\mu_{i} \mathbf{r}_{i j}\right)\left(\mu_{j} \mathbf{r}_{i j}\right)\right],
$$

where $\mathbf{r}_{i j}=\mathbf{r}_{j}-\mathbf{r}_{i}$. One of the features of the dipolar interaction is its long range nature. For a three dimensional dipolar system, the interaction is strictly long range because of the

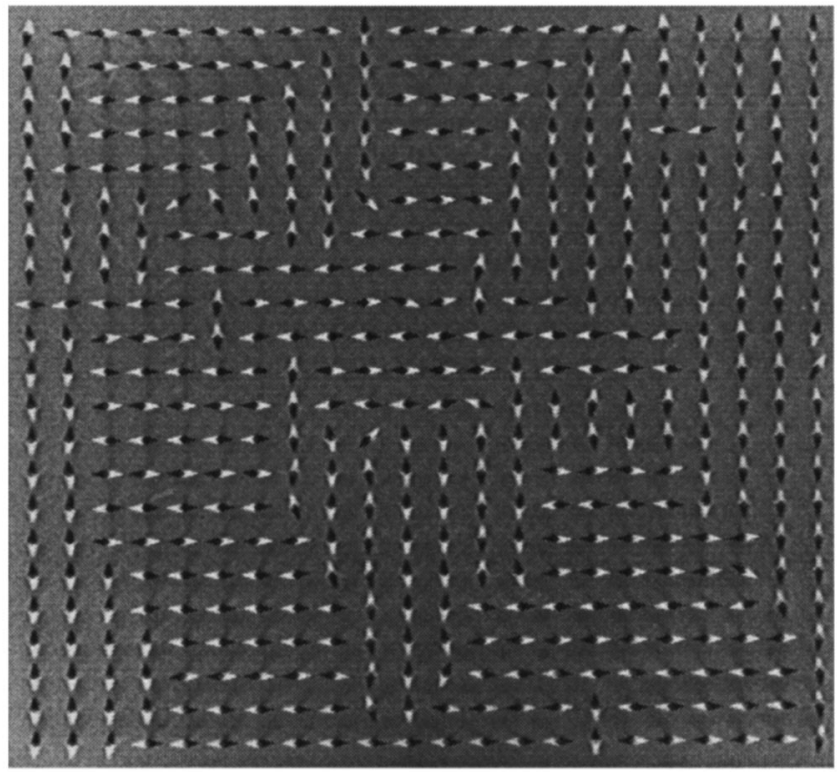

FIG. 2. Example of an experimental trapped configuration. When the system is disordered with a field of random amplitude and direction (see Fig. 1), then switching off quickly this excitation leads the system to such a metastable state. 


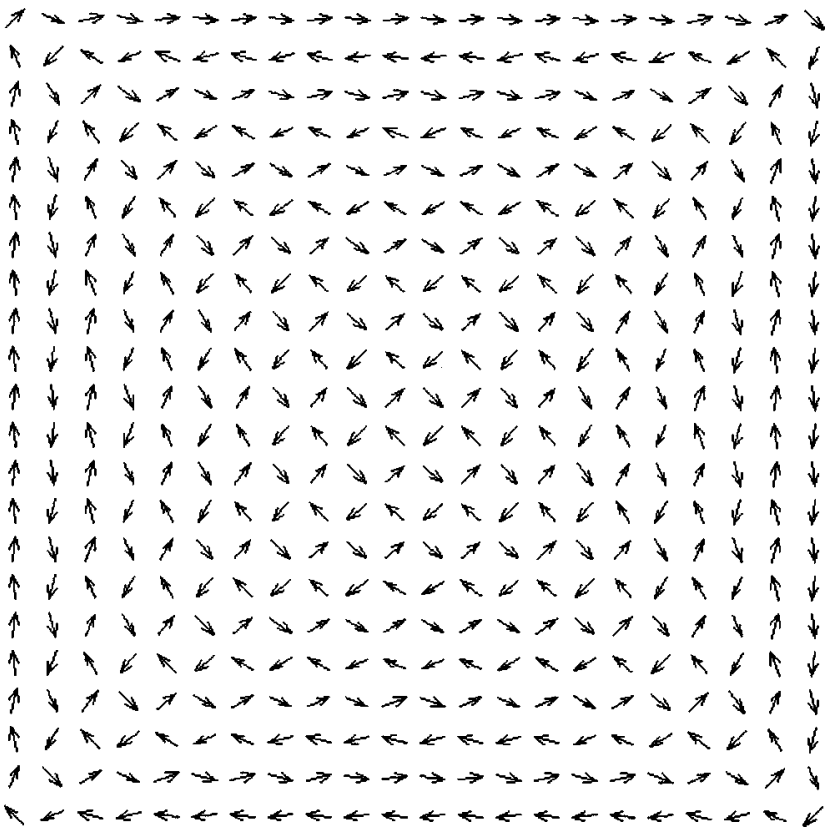

FIG. 3. Ground state of a $22 \times 22$ square lattice of "pure" dipoles obtained by numerical simulation. Lines of dipoles are observed on the edges while in the center symmetry reasons lead to a microvortex structure. The finite size effects remove the continuous degeneracy which exists in the infinite square lattice. As described in Sec. II, such a microvortex configuration cannot exist in the $22 \times 22$ experimental compass needle lattice because of the multipolar terms effects.

total energy divergence. In the case of 2D dipolar system, the interaction is strictly speaking not long range anymore since the total energy converges. However the long range denomination is still used to describe the slow algebraic decay of this interaction compared to the usual exponential decay of the exchange interaction.

The dipolar interaction has also an anisotropic feature which means that, in addition to the relative orientation of the magnetic moments (isotropic term $\boldsymbol{\mu}_{i} \boldsymbol{\mu}_{j}$ ), the interaction depends on their orientation with regard to the vector $\mathbf{r}_{i j}$ [anisotropic term $\left(\boldsymbol{\mu}_{i} \mathbf{r}_{i j}\right)\left(\boldsymbol{\mu}_{j} \mathbf{r}_{i j}\right)$ ]. Thus the minimization of the dipolar energy has to satisfy both aspects which leads to the natural notion of lines of dipoles. However, in spite of this anisotropic feature, the ground state of an infinite square lattice of "pure" dipoles, which is described by two antiferromagnetic sublattices, has a continuous degeneracy. $7,9,12,13$ If we consider a finite square lattice of such "pure", dipoles, then the ground state is not continuously degenerated anymore and dipoles form lines on the edge. In the case of a pure dipole lattice of the same $22 \times 22$ size as our experimental lattice, symmetry reasons lead to a microvortex structure in the center of the lattice (Fig. 3).

We shall describe now the particular case of the compass needle lattices and explain why such a microvortex structure cannot be observed in this system. Since the size of the compass needles $(10 \mathrm{~mm})$ is not small compared to the lattice spacing $(12 \mathrm{~mm})$, the dipolar approximation is obviously insufficient to describe the interaction between the compass needles. It is thus advisable to take into account higher order terms. Their main effect is to reinforce the anisotropic feature of the dipolar interaction by reducing to four the pos- sible orientations for each compass needle. This anisotropic effect of the multipolar terms is shown by Erber ${ }^{14}$ and Klymenko. ${ }^{15}$ This degeneracy breaking by the multipolar terms which select the square lattice axes induces the stabilization of a long range order. Klymenko equally showed a long range order stabilization in diatomic polar molecules by the quadrupole interaction. In our case, due to the rhombic shape of the compass needles, the quadrupolar terms cancel each other and the first multipolar terms are the octupolar ones.

Hence, as a result of these octupolar terms, the system can be trapped into metastable states such as the one shown in Fig. 2. They also justify that such a configuration shown in Fig. 3 occurs only in the "pure" dipolar lattice, and can not exist in the compass needle lattice where the ground state is made of antiparallel lines of compass needles along the lattice axes.

\section{EXPERIMENTS AND NUMERICAL SIMULATION}

Until now, we have described in details our experimental system, describing some of its behaviors and pointing out its specificity compared to a purely dipolar lattice. We now present the study where this $22 \times 22$ square lattice of compass needles is subjected to an applied field of random direction and amplitude chosen between 0 and $H_{\max }$. The frequency of this random field, i.e., the rate of change of $H$ in the range $\left[0, H_{\max }\right]$, is close to resonant frequencies of this dissipative dynamic system (about $8 \mathrm{~Hz}$ ). As already said, we observe oscillations of the needles, weakly disruptive for the lines of needles for small values of $H_{\text {max }}$, and larger oscillations or chaotic rotations with increasing $H_{\text {max }}$ leading to short line lengths with short lifetimes. Thus, observing the lattice behaviors while increasing $H_{\max }$, leads to a straightforward analogy in which the system exhibits a phase transition between an ordered phase and a disordered one, and where the relevant excitations tend to destroy the lines of compass needle. In this analogy, the fluctuations induced by the random field represent the thermal fluctuations. Therefore, we want in the following to make a thermodynamic approach of this dynamic dissipative system in order to test such an analogy. In this approach, the upper limit value $H_{\max }$ of the random field will define a "temperature" scale.

Generally, in order to simulate thermal fluctuations on a dynamic system one may apply a random force. Although such a mapping of a dynamic system onto equilibrium statistical mechanics remains generally unsolved, it can be shown that under the condition of "detailed balance" the mapping is successful (see for example Ref. 16). The random field we apply is of random amplitude and direction and hence, uncorrelated at different times, but there exist spatial correlations for this applied random field which is uniform at each pulse. However, it is important to note that the relevant quantities in the equation of movement are the torques, e.g., $\boldsymbol{\mu}_{i} \times \mathbf{H}$, where $\mathbf{H}$ is the random field. Therefore the random field which is uniform at each pulse, induces spatially uncorrelated torques due to the slight distribution of both needles magnetization and friction. Hence, we assume that the weak experimental inhomogeneities restore this spatially uncorrelated aspect.

In the approach we want to develop, the fluctuations due 
to the applied random field represent the thermal fluctuations. Thus, in order to compare both meanings of the temperature, the experimental results will be compared to a Monte Carlo numerical simulation adapted to the experimental system. In this simulation, the system is not subjected to a random field but to the thermal fluctuations following the Boltzmann statistics. The Hamiltonian which describes the compass needle interaction contains the dipolar term written in Eq. (1). In this simulation we choose free edge boundary conditions and we take into account all the neighbors to sum the pair energies, since it is well known that, as opposed to the uniform long range interaction case, a mean field treatment is uncertain and sometimes inaccurate for anisotropic long range interactions such as the dipolar interaction (see for example Refs. 17 and 18).

As already said, the dipolar approximation is not sufficient to describe the compass needle interaction and higher order terms are needed. In order to simplify the numerical calculations, these terms are not explicitly written but are replaced by a simpler one which accounts for their biaxial anisotropy effects described in Sec. II:

$$
H_{\mathrm{ani}}=k \sum_{i} \mu_{i}^{2} \sin ^{2}\left(2 \theta_{i}\right)
$$

where $\mu_{i}$ are the magnetic moments and $\theta_{i}$ their angle with the lattice axes. The constant $k$ allows to adjust the strength of the anisotropy in order to reproduce the experimental behaviors, like the reversal of magnetization by avalanches. The best value that we found is when $\left(k \mu^{2}\right) /\left(\mu^{2} / a^{3}\right)=1$, where $a$ is the lattice spacing which is a constant of the system.

We have run the usual Monte Carlo method using the Metropolis algorithm (for details see for example Ref. 19). We defined the MCS ("Monte Carlo step") time unit as $2 N^{2}$ random trials for the $N \times N$ square lattice. We have computed up to $10^{5}$ MCS for a given temperature and to be sure that transients have died, the first MCS were discarded and not used in computing averages of equilibrium thermodynamic quantities. Several lattice sizes from $10 \times 10$ up to $28 \times 28$ compass needles have been computed.

With regard to the experiments, the procedure is as follows. A strong random field is applied to the lattice of compass needles in order to disorder it. $H_{\max }$ is then slowly decreased down to the value we want to study. When the equilibrium is supposed to be reached (see Sec. IV A), the measurements begin. The image sampling frequency is 0.25 $\mathrm{Hz}$. These four seconds are needed to analyse and store the $22 \times 22$ compass needle angles. The experiment duration is two hours which gives 1800 configurations of the system for a given value of $H_{\max }$.

\section{EXPERIMENTAL AND NUMERICAL RESULTS}

\section{A. Line length}

We have mentioned in the first observations of the experimental system described in Sec. II that decreasing $H_{\max }$ very slowly from a disordered state gives a configuration which is close to the ground state, i.e., we observe an antiparallel line configuration but in some places parallel lines can be formed since there is a strong line structure which traps the system into metastable states. The main feature of such states is the existence of lines whether they are parallel or antiparallel. Therefore, we will consider that for a given value of $H_{\max }$ the configurations are characterized by the line lengths. Although parallel and antiparallel lines do not have exactly the same energy, we observe that we can define an equilibrium average line length for a given value of $H_{\max }$. It means that for different initial conditions at a given value of the random field, the system may be trapped in slightly different energy minima but all are characterized by the same average line length and the same fluctuations around this value. The effect of the random field is to break this line structure by reducing their average size. This description in terms of lines of needles allows us to consider both the very stable "ferromagnetic" configuration and the ground state configuration (antiparallel lines) as ordered states.

To define lines at a given value of the random field in the experiment or at a given value of the temperature in the Monte Carlo simulation, each of the compass needles of a given configuration is virtually folded back to the nearest crystallographic axis. A needle is in the same line as its neighbor if both are aligned with $\mathbf{r}_{i j}$ which join them, and in the same direction. If they are aligned with $\mathbf{r}_{i j}$ but in the opposite direction or if they are perpendicular, then they take part in two different lines. Each needle counts as a unit length. Since the lines can equally form parallel or antiparallel configurations, we will focus on the average line length in the perpendicular $x$ and $y$ directions. Note that in the numerical simulation this four state discretization procedure, which allows the computation of the average line lengths of compass needles in the two perpendicular $x$ and $y$ directions, is not equivalent to a four-state model since we allow here all possible orientations for the needle angles between 0 and $2 \pi$ during the calculations. The discretization procedure to four states only takes place to compute the line length.

The typical evolutions of the equilibrium line lengths $l_{x}$ and $l_{y}$ for the $22 \times 22$ lattice are displayed versus time for three different values of $H_{\max }$ in experiments (Fig. 4) and for three different temperatures in the Monte Carlo simulation (Fig. 5).

It is obvious that at low values of $H_{\max }$ shown in Fig. 4(a), and low temperatures shown in Fig. 5(a), one of the $x$ or $y$ directions is favored in the sense that needle lines are preferentially formed in this direction (larger line length). The $x$ and $y$ directions are equivalent $a$ priori and one of them is chosen by the system according to the initial configuration. If one of the direction is initially favored the system will then keep this alignment on average. If none of the direction is initially favored the system will then be guided to one of them by the fluctuations. Thus, at low values of $H_{\max }$ in the experiments and at low temperatures in the Monte Carlo simulation, we clearly define the existence of a preferential direction which according to the initial configuration can be the $x$ or $y$ direction.

On the contrary one can see that, for higher amplitude of the random field shown in Fig. 4(c), and higher temperature in the Monte Carlo simulation shown in Fig. 5(c), there is no longer a preferential direction in the system since at time scale needed to define an average line length (at least few seconds and few MCS), both average line lengths $\left\langle l_{x}\right\rangle$ and $\left\langle l_{y}\right\rangle$ are equal. 
(a)

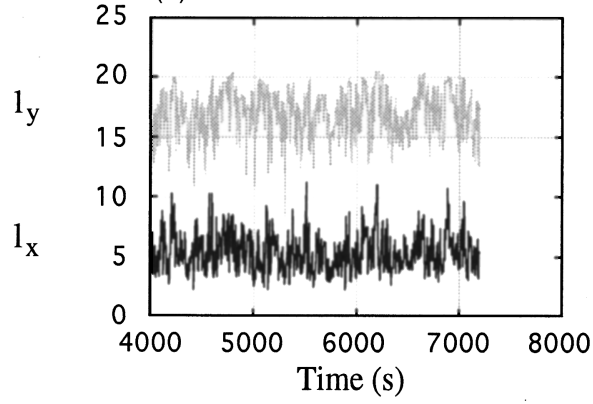

(b)

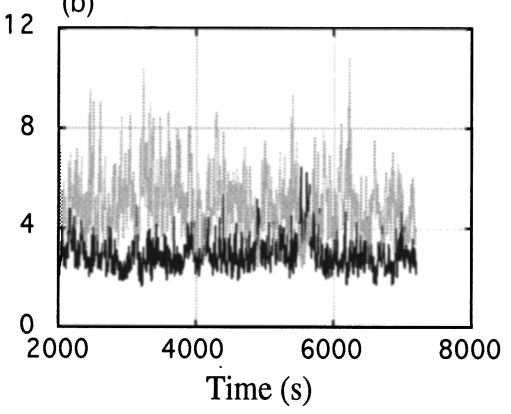

(c)

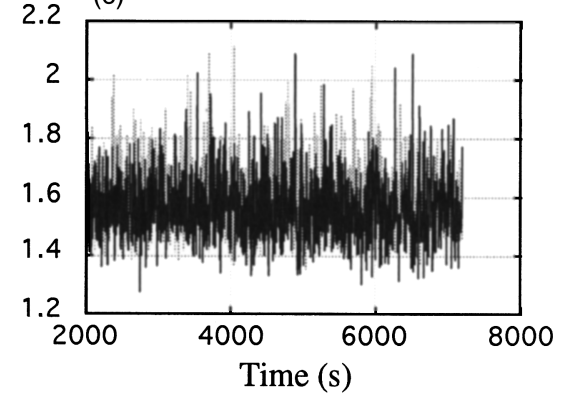

FIG. 4. Experimental results $\left(22 \times 22\right.$ lattice) for the equilibrium line lengths $l_{x}$ and $l_{y}$ versus time, for three different values of $H_{\max }$ : (a) below the critical value of $H_{\max }$; (b) near the critical value of $H_{\max }$; (c) above the critical value of $H_{\max }$.

Hence, we observe a transition from a state defined by the existence of a preferential direction, to an isotropic one where no privileged direction can be defined. Therefore, the order in this system should be characterized by the emergence of a preferential direction. Furthermore, when the system is close to this transition, one can see in Fig. 5(b) that the thermal fluctuations in the Monte Carlo simulation allow the system to change in time its preferential direction. Despite this alternation, this figure shows that the favored direction notion is still meaningful since this direction remains the preferential one during time scale larger than the MCS time unit. This alternation phenomenon is less clear in experiments, see Fig. 4(b), but it exists, see for instance at $t$ $\approx 5500 s$. Probably the time scale needed for this alternation phenomenon is larger than the experiment durations.

In conclusion, when decreasing the amplitude of the random field in the experiments (or when cooling the system in the Monte Carlo simulation), from a disordered isotropic phase without favored direction, a broken symmetry arises inducing the emergence of a preferential direction. Besides this emergence of a preferential direction, there is a second aspect which characterizes this transition, namely, an average line length increase as seen in Fig. 4 and Fig. 5. This aspect will be discussed in Sec. IV C.

\section{B. Order parameter, susceptibility, and specific heat}

Following the previous description, the order is characterized by the emergence of a preferential direction. Since in a given direction parallel and antiparallel lines are assumed to be equivalent, it is straightforward to define the order param- eter which breaks the symmetry. One only has to count the compass needles in both $x$ and $y$ directions, namely $n_{x}$ and $n_{y}$. The difference between $n_{x}$ and $n_{y}$ should then express whether one direction is more "populated" than the other. Besides, the favored direction may change in time near the transition. This alternation should not affect the order parameter which must keep on expressing the existence of a favored direction. Thus, the absolute value of the difference $\left|n_{x}-n_{y}\right|$ allows to preserve this information. The order parameter defined in this way is written:

$$
q=\frac{1}{N^{2}}\left\langle\left|n_{x}-n_{y}\right|\right\rangle,
$$

where $N^{2}=n_{x}+n_{y}$ is the total number of needles. The order parameter is plotted versus $H_{\max }$ in Fig. 6(a) for the experimental system, and versus the normalized temperature $T^{*}$ $=k_{B} T /\left(\mu^{2} / a^{3}\right)$, where $a$ is the lattice spacing, in Fig. 6(b) for the Monte Carlo simulation.

In both experiment and numerical simulation, due to the finite size effects, the order parameter is not equal to one at finite temperature below the transition. Indeed, in order to minimize the system energy, the compass needles along the edge which is perpendicular to the preferential direction, form a transverse line parallel to the edge. As a result, the order parameter decreases. Above the transition, one can see from the Monte Carlo results that the larger the lattice size, the faster the decrease of the order parameter, showing that it would be zero for an infinite system. Nevertheless, the observed order parameter tails contain a well known unphysical aspect. $^{20}$ At high temperature, the finite system is disordered
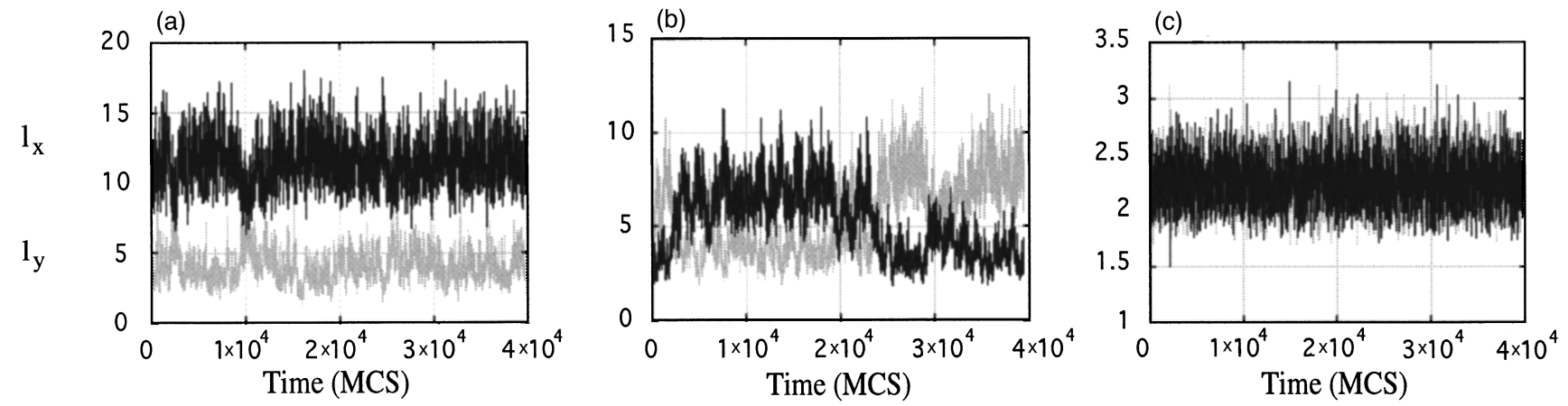

FIG. 5. Monte Carlo simulation results $(22 \times 22$ lattice $)$ for the equilibrium line lengths $l_{x}$ and $l_{y}$ versus Monte Carlo Step, for three different temperatures: (a) below the critical temperature; (b) near the critical temperature; (c) above the critical temperature. 


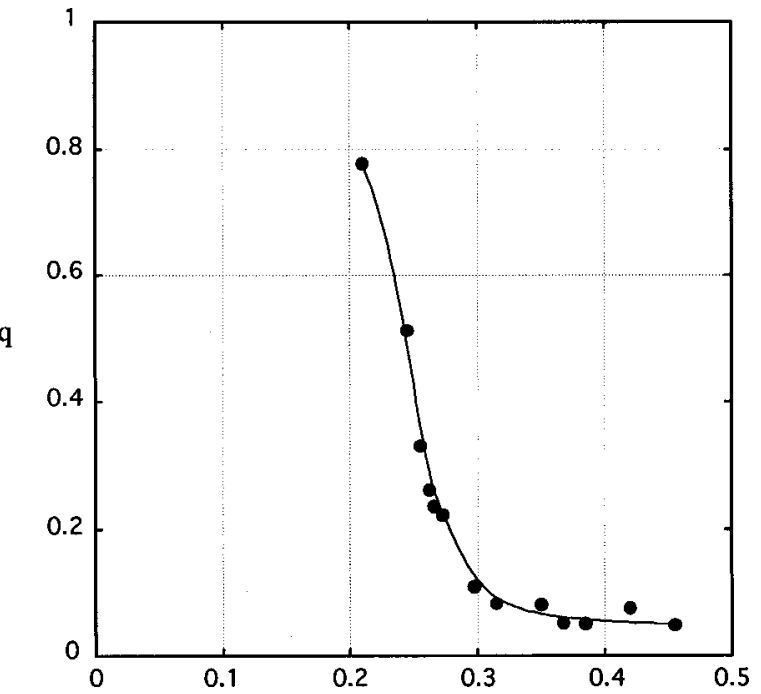

(a) $\mathrm{H}(\mathrm{Oe})$

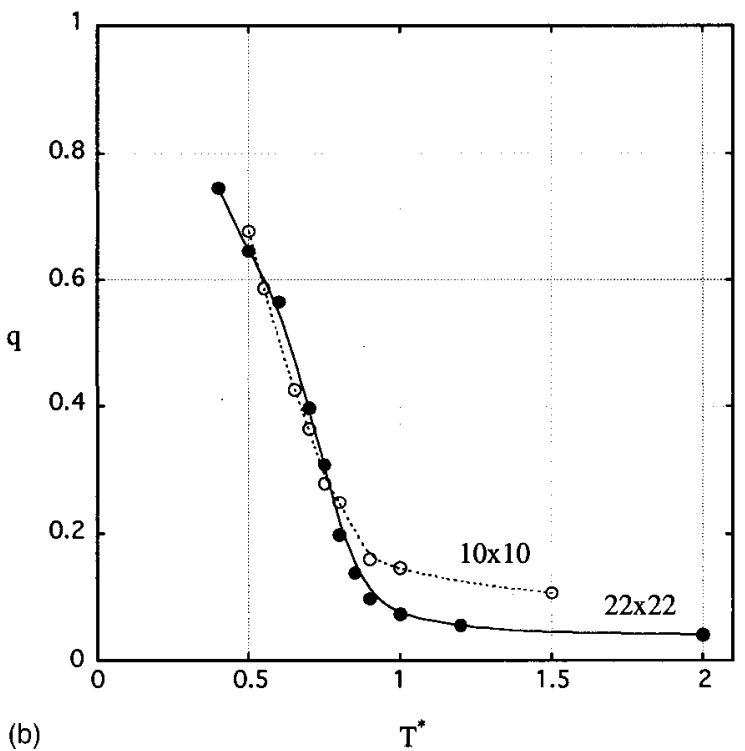

FIG. 6. Order parameter: (a) versus the amplitude $H_{\max }$ for the experimental $22 \times 22$ lattice. This applied random field is supposed to mimic the thermal fluctuations; (b) versus temperature for two lattice sizes in the Monte Carlo simulation.

since $n_{x}$ and $n_{y}$ have identical fluctuations around the same average value $\left\langle n_{x}\right\rangle=\left\langle n_{y}\right\rangle=N^{2} / 2$. But the absolute value of the difference $\left|n_{x}-n_{y}\right|$ fluctuates around a nonzero value. That is the reason for the unphysical nonvanishing order parameter observed at high temperature.

The directional aspect which characterizes the transition reminds what occurs in nematic liquid crystals. By analogy we call director the preferential direction. The 2D nematic order parameter is defined as (see for instance Refs. 21 and 22)

$$
q_{\text {nematic }}=2\left\langle\frac{1}{m} \sum_{i} \cos ^{2} \theta_{i}\right\rangle,
$$

where $m$ is the number of molecules and $\theta_{i}$ their angle with the director. In the case of our compass needles lattice we defined the director as the $x$ or $y$ direction. Let $n^{*}$
$=\operatorname{Max}\left(n_{x}, n_{y}\right)$ be the number of needles aligned with the director. The order parameter defined in Eq. (2) can then be written:

$$
q=\frac{1}{N^{2}}\left\langle n^{*}-\left(N^{2}-n^{*}\right)\right\rangle=\frac{2}{N^{2}}\left\langle n^{*}\right\rangle-1 .
$$

The order parameter that we have defined is thus analogous to the one defined for the isotropic-nematic phase transition. It is also with a nematic analogy that Romano ${ }^{18}$ computed the order parameter for a purely dipolar square lattice. In that paper, the director was not restricted to the $x$ and $y$ directions. The critical temperature we deduced with our procedure in the numerical simulation, is in good agreement with Romano who found a slightly smaller value due to the absence of multipolar terms.

The susceptibility associated to the order parameter, computed via the fluctuations of $\left|n_{x}-n_{y}\right|$ is

$$
\chi_{q}=\frac{1}{N^{2}} \frac{\left\langle\left|n_{x}-n_{y}\right|^{2}\right\rangle-\left\langle\left|n_{x}-n_{y}\right|\right\rangle^{2}}{T^{\prime}}
$$

where $T^{\prime}$ is either $H_{\max }$ in the experiments, or the normalized temperature $T^{*}=k_{B} T /\left(\mu^{2} / a^{3}\right)$ in the Monte Carlo simulation. Both susceptibilities defined in the experiments [Fig. 7(a)] and in the numerical simulation [Fig. 7(b)] display a maximum which confirms the existence of a phase transition.

Equilibrium states are meaningful in numerical simulations since the Monte Carlo technique allows to reach them on reasonable time scales. But in the experiments the system may be trapped in configurations which are close to the minimum of free energy and time scales needed to reach the real equilibrium state are too large. Nevertheless, we have defined a relevant quantity, namely the average line length, which reaches a well defined equilibrium value even if the system is trapped in slightly different energy minima. Therefore, since the energy of the system computed in the experiments does not always correspond to the equilibrium (especially at low amplitude of the random field), we do not display results of the experimental specific heat but only for the Monte Carlo numerical simulation. The specific heat is computed, via the fluctuation-dissipation theorem, as follows:

$$
C_{V}=\frac{\left\langle E^{2}\right\rangle-\langle E\rangle^{2}}{k_{B} T^{2}}
$$

where $E$ is the internal energy of the system, $T$ is the temperature and $k_{B}$ is the Boltzmann constant. The specific heat, normalized by $N^{2} k_{B}$, is plotted in Fig. 8 for several lattice sizes versus the normalized temperature $T^{*}=k_{B} T /\left(\mu^{2} / a^{3}\right)$. Figure 8 shows maxima which become sharper when the lattice size is increased. Besides, we verify the shift of the critical temperature which increases with the lattice size. This is what is to be expected for free edge boundary conditions in spins lattices with exchange interactions. ${ }^{23} \mathrm{We}$ also verify the rounding of the specific heat in the temperature range [0.7-0.9], which is due to the finite size effects (see for instance Refs. 24 and 25). Here the fluctuation- 

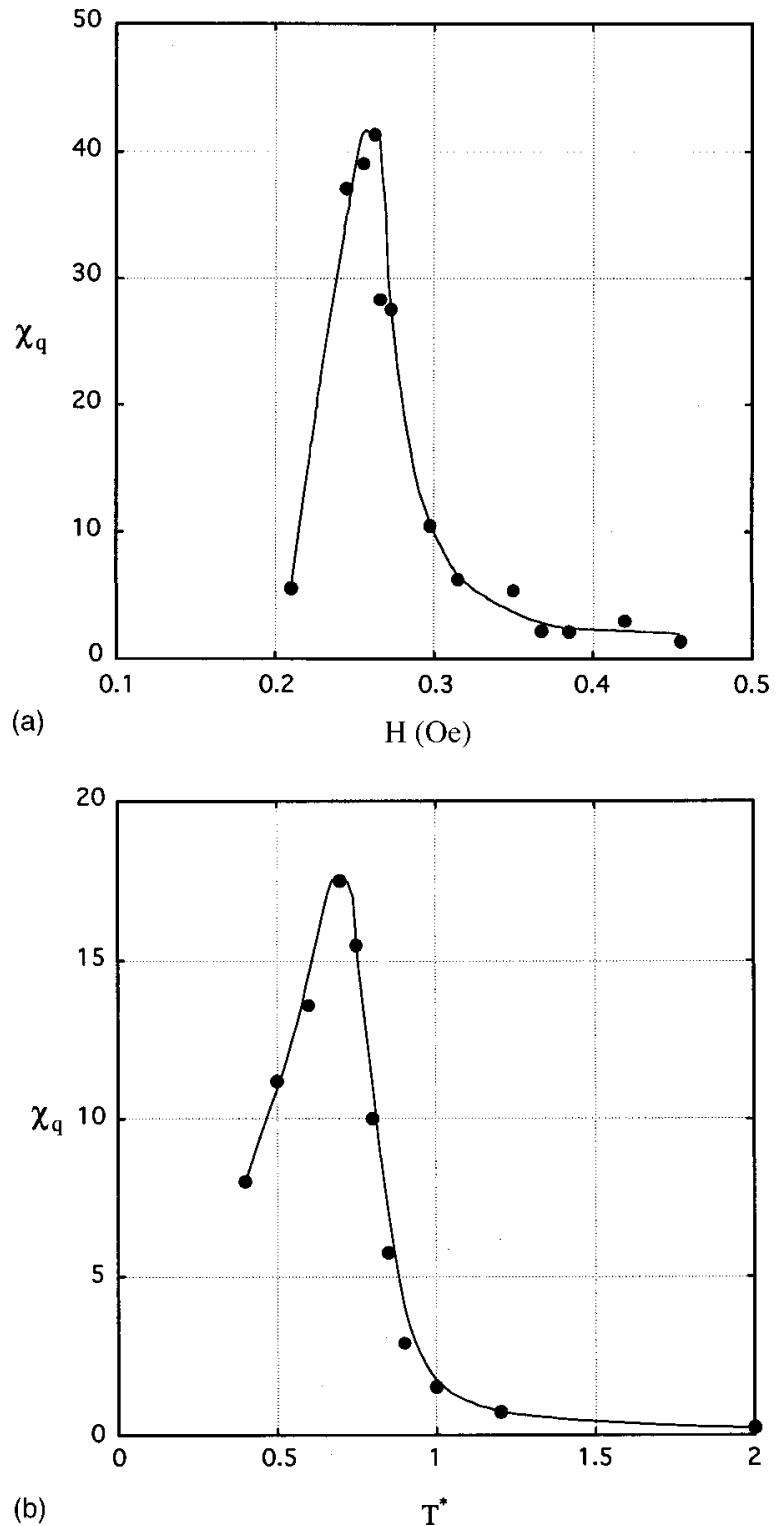

FIG. 7. Susceptibility associated to the order parameter for the $22 \times 22$ lattice; (a) versus the amplitude $H_{\max }$ for the experimental lattice; (b) versus the temperature in the Monte Carlo simulation.

dissipation theorem is not valid anymore since the correlation length becomes of the same order of magnitude as the size of the system.

The presence of specific heat maxima, increasing with the lattice size and which seems to lead to a divergence, confirms the existence of a phase transition revealed both in the experiments and in the numerical simulation by the order parameter susceptibilities.

\section{C. "Defects" in the making of lines}

We have shown that the phase transition was characterized by two aspects. The first one deals with the emergence of a director when decreasing the amplitude of the random field through a critical value or when cooling the system in the Monte Carlo simulation through the critical temperature. We have defined the order parameter which breaks the isotropy of the disordered phase. The second aspect which characterizes this transition is the line length increase with de-

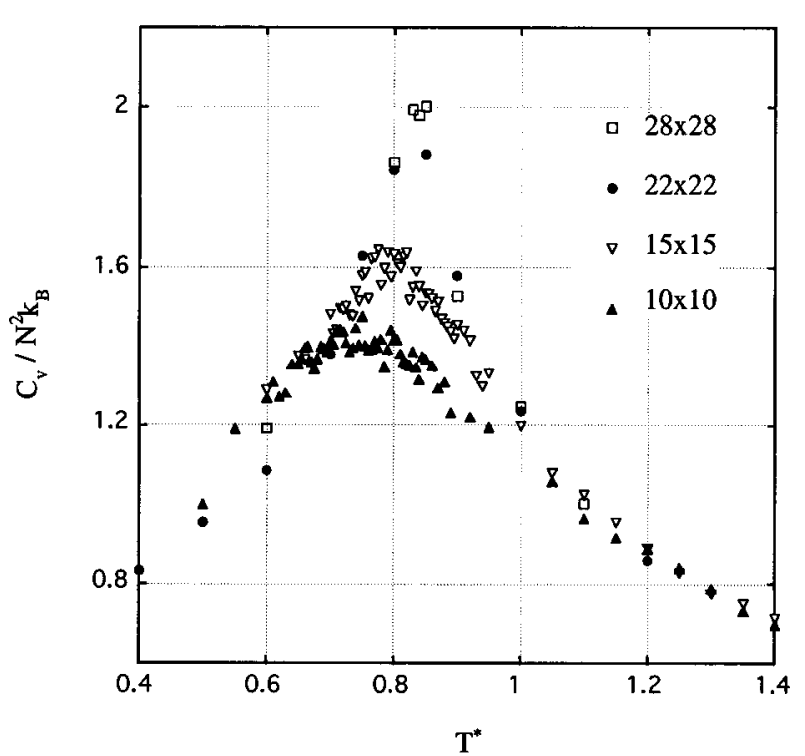

FIG. 8. Monte Carlo simulation results for the specific heat computed from the internal energy fluctuations for several lattice sizes. The presence of specific heat maxima, increasing and becoming sharper with the lattice size confirms the existence of a phase transition.

creasing $H_{\max }$ or the Monte Carlo temperature. In order to describe more precisely this second aspect of the transition, we introduce a new quantity which is closely related to the line length. Instead of describing the system in terms of lines, we describe it in terms of lines disruptions which means that we are now interested in the bonds of the square lattice. In Sec. IV A, we have considered an analysis procedure where each needle is folded back to the nearest crystallographic axis to compute the line length. This means that we defined $4 \times 4=16$ possible states for a bond which links two needles. These states and their degeneracy are shown in Fig. 9. The six states shown in Fig. 9(a) describe bonds that do not disrupt lines of needles whereas the ten states shown in Fig. 9(b) do. These latter bonds are called defects in the sense that they are defects in the making of lines.

For a given configuration, we are now interested in the number of defects $d$ among the $B=2 N(N-1)$ bonds of the $N \times N$ square lattice. We can describe the equilibrium states of the system in terms of equilibrium number of defects $\langle d\rangle$ which means that for a given value of $H_{\max }$ in experiments or for a given temperature in the Monte Carlo simulation, the system is characterized by an average number of defects and

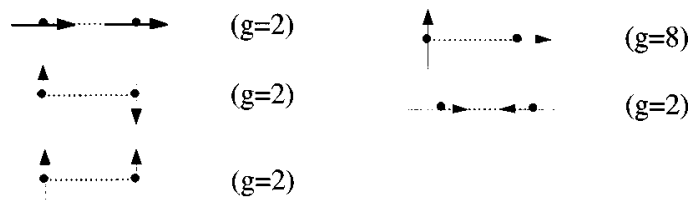

(a)

(b)

FIG. 9. Possible states (and their degeneracy $g$ ) for a bond of the square lattice when each compass needle is folded back to the four axes. The bonds in (a) do not disrupt lines of needles. On the contrary, the bonds in (b) disrupt lines of needles and are therefore called defects. 
by fluctuations around this value. The ground state is a zero defect structure. The completely disordered configuration is not characterized by $\langle d\rangle=B$ defects because of entropy considerations. Indeed, the only way to have this number of defects is when each needle is perpendicular to its four nearest neighbors. Thus, there is only a small number of such states. We can see that among the sixteen states in Fig. 9, ten are defined as defects, which gives a probability for a bond to be a defect of $5 / 8$. Thus, when the correlations between the needles disappear, $\langle d\rangle=5 B / 8$. Hence, the maximum of entropy, as a function of $d$ is obtained for $\langle d\rangle=5 B / 8$. If $\langle d\rangle$ $<5 B / 8$, then the fluctuations statistically create defects and when $\langle d\rangle>5 B / 8$ then the fluctuations statistically annihilate defects.

We can now define a parameter $\delta$ which is expected to vary from one in the ordered state, to zero in the completely disordered one

$$
\langle\delta\rangle=1-\frac{8}{5 B}\langle d\rangle .
$$

The evolution of $\langle\delta\rangle$ is shown for the experiments in Fig. 10(a) versus $H_{\max }$, and for the Monte Carlo simulation in Fig. 10(b) versus the temperature. The data computed in the numerical simulation show that the different curves corresponding to several lattice sizes tend to converge in a unique curve above the critical temperature. Thus, the number of defects per bond becomes nearly independent of the lattice size above the transition. Furthermore, even at very high temperature, the system does not reach its maximum of entropy which means that there are still remaining correlations that prevent the system from being completely disordered. Hence, the defect variable shows that even far above the transition there is a remaining short range order.

\section{DISCUSSION}

This experimental and numerical study of a square lattice of compass needles showed the existence of a phase transition between an ordered phase and a disordered one. We defined relevant quantities such as the average line lengths of the compass needles in the perpendicular $x$ and $y$ directions. These quantities allow to give a detailed description of the observed transition which is characterized by the emergence of a preferential direction, and by an increase of the average line length. The first aspect describes a broken symmetry and the associated order parameter has been defined. Furthermore, a description in terms of lines disruptions (called defects) showed that above the transition the system is not completely disordered showing a remaining short range order.

All the results presented in this paper displayed good qualitative agreements between the experiment and the numerical simulation. However, both have their own approximation level. On one hand, the experimental lattice of compass needles is a complicated dynamic magnetic system. The velocities and dissipation are not taken into account in the configurations analysis, but only the needles angles which are measured by regular snapshots. On the other hand, the interaction Hamiltonian of the same system is analyzed by an usual Monte Carlo numerical simulation. The energy is approximately known because of the multipolar terms which
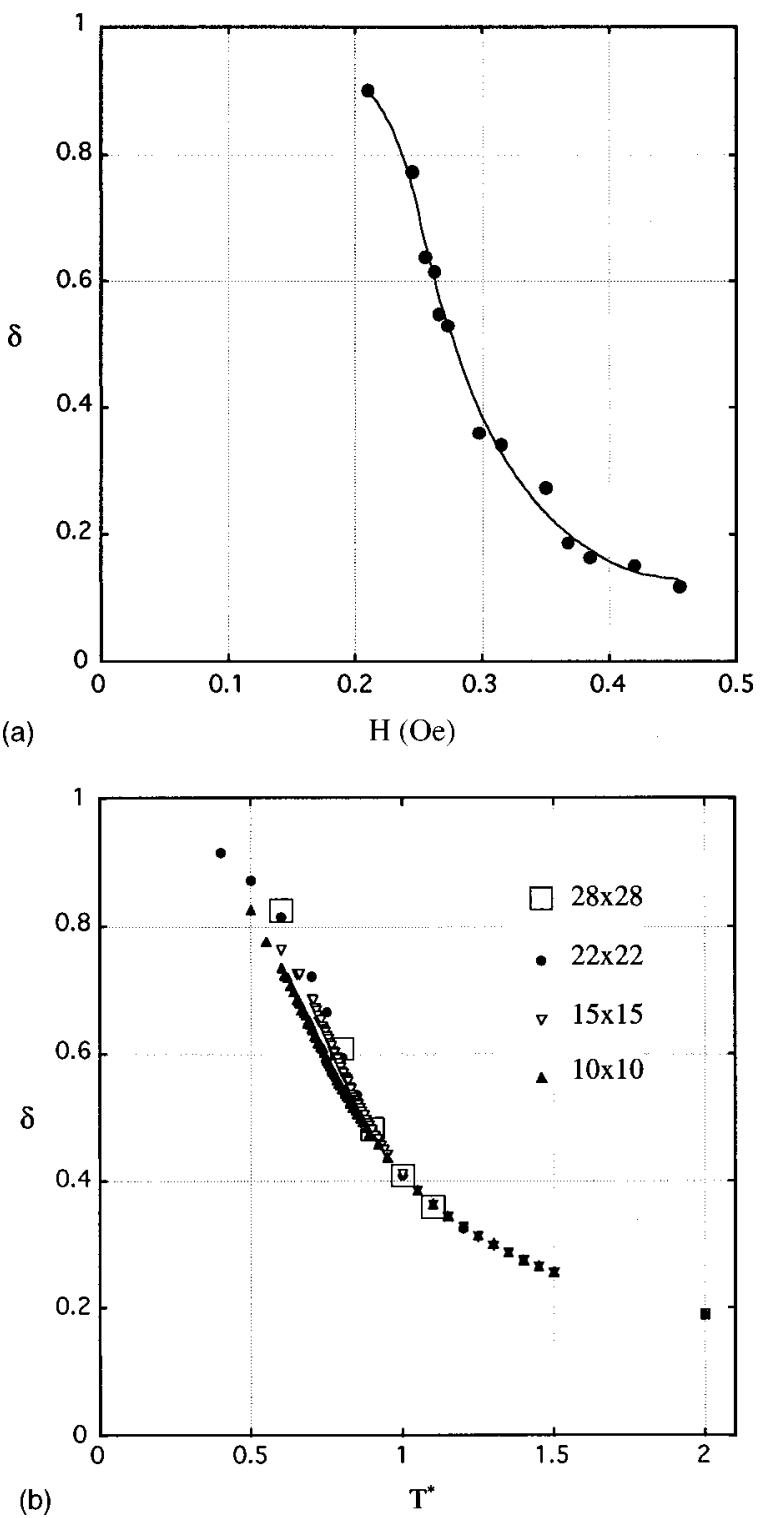

FIG. 10. Evolution of the $\delta$ parameter: (a) versus the amplitude $H_{\text {max }}$ for the experimental $22 \times 22$ lattice; (b) versus the temperature in the Monte Carlo simulation for the several lattice sizes. $\langle\delta\rangle=1$ $-8\langle d\rangle / 5 B$, defined with the defect variable, is such that $\delta=1$ in the ordered state (zero defect), and $\delta=0$ in the completely disordered state (maximum number of defect $\langle d\rangle=5 B / 8$ ).

are reduced to a simple biaxial anisotropy term. Furthermore, the temperature has two meanings. In the Monte Carlo simulation the temperature allows the system to probe the phase space according to the Boltzmann statistics whereas in the experiments thermal fluctuations are simulated by an applied random field. The mapping of a dynamic dissipative system onto equilibrium statistical mechanics is a crucial problem which leads to fascinating fundamental questions. The results presented in this paper show a successful example of such a mapping.

Beyond the good qualitative agreement observed between the experiment and the numerical simulation, we now want to compare the results in a quantitative way in order to bring more evidence to this successful mapping. To compare the results we need to renormalize the applied random field. A theoretical approach to associate a temperature to each value 


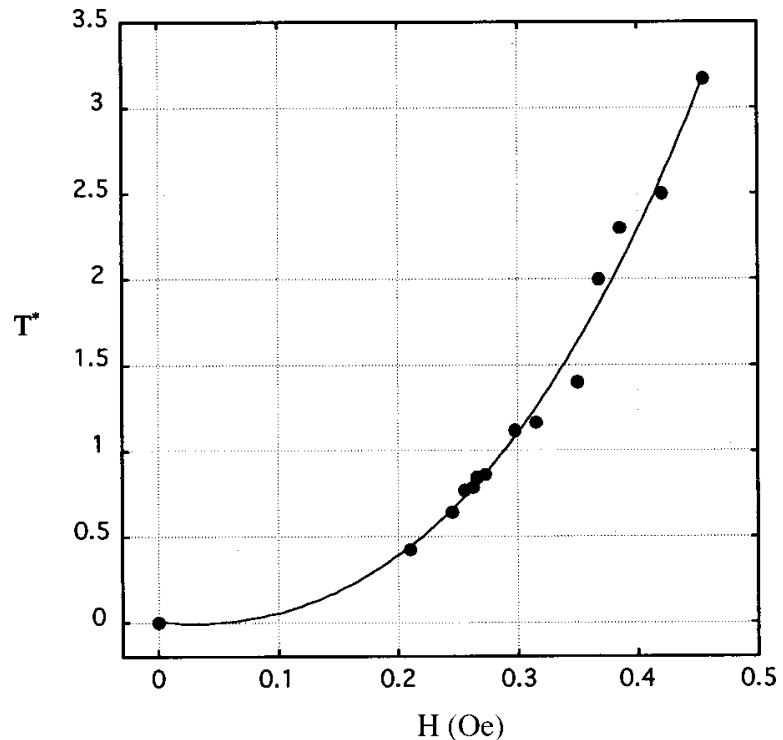

FIG. 11. Variation of the Monte Carlo reduced temperature $T^{*}$ as a function of $H_{\max }$, maximum value of the applied random field, for the $22 \times 22$ lattice size. Assuming that the applied random field gives rise to fluctuations analogous to the thermal fluctuations, this variation is deduced from the superposition of the $\delta$ parameter curves of the experiments and the Monte Carlo simulation (see Fig. $10)$.

of $H_{\max }$ is not straightforward for this complicated dissipative dynamic system. But, in some sense we can have a measure of this renormalization. Indeed, imposing a superposition between the experimental and numerical results for the the $\delta$ parameter, allows us to determine the temperature associated to each value of the applied random field which mimics the thermal fluctuations. This leads to a nonlinear evolution of the Monte Carlo temperature versus $H_{\max }$ shown in Fig. 11. We can now compare the experiment and the numerical simulation for the order parameter, shown in Fig. 12(a), and for the order parameter susceptibility, shown in Fig. 12(b). Thus, as one can see, a satisfactory agreement is obtained.

Therefore, we are able to find a good agreement between a thermodynamic analysis of a dissipative dynamic system excited by a random field, and the numerical simulation of the hamiltonian of the same system treated by an usual Monte Carlo technique. This good agreement assumes a nonlinear variation of our "experimental temperature" as a function of the applied random field. Understanding such a variation is not easy because of the complicated and highly nonlinear dynamics of this system, and further studies should be necessary. In particular, an additional numerical simulation where the compass needle lattice is subjected to a random field, as in the experiments, would be useful and interesting to compare with our results. One could study in this way the effect of a spatial disorder which has to be introduced in the system, since the applied random field is uniform at each pulse, in order to restore the spatially uncorrelated aspect which is necessary for a successful mapping. One could see for example the effect of a small spatial distribution of the magnetic moments. Such a simulation is a
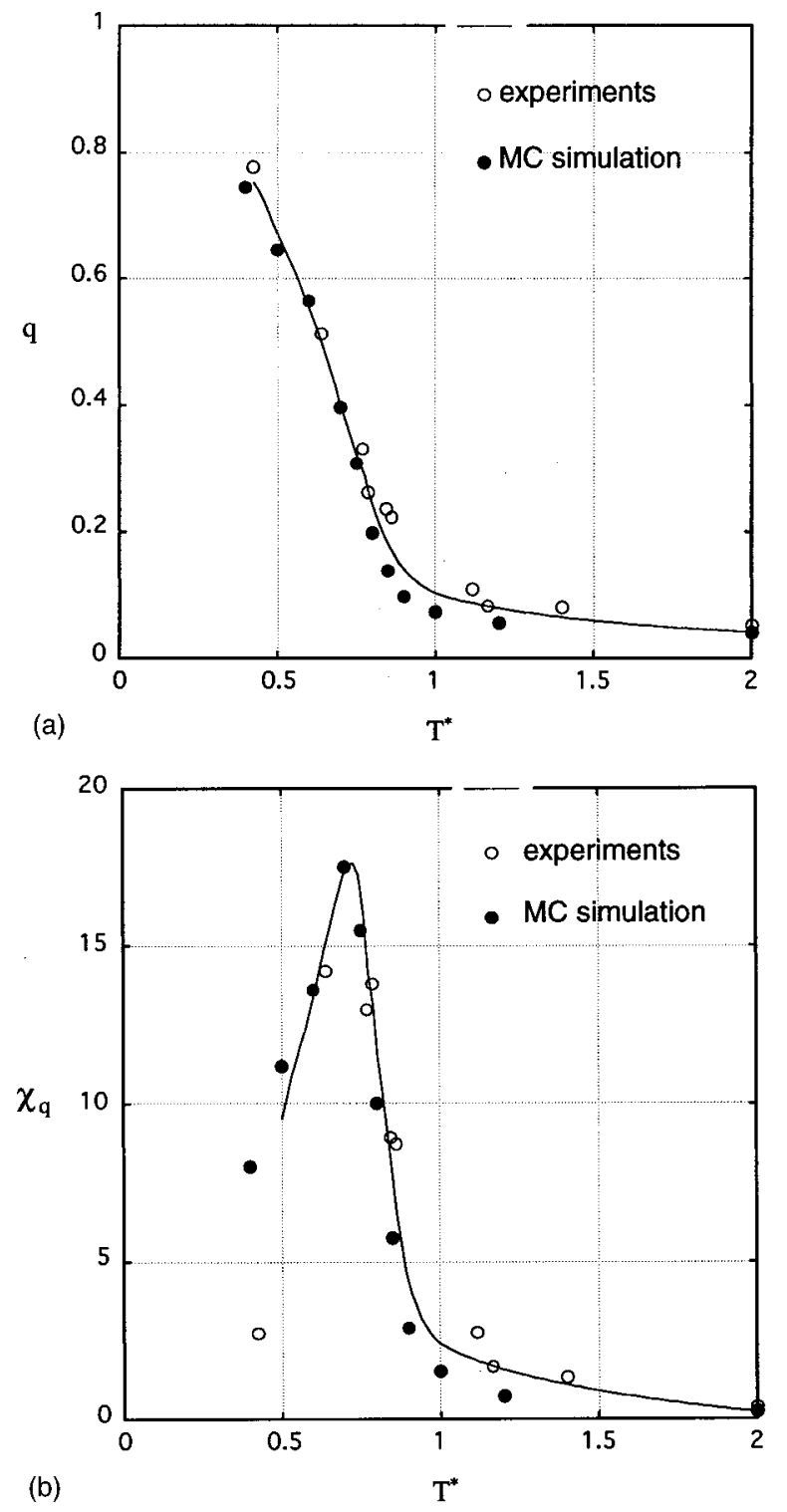

FIG. 12. Comparison between experimental (open circles) and Monte Carlo numerical results (filled circles) for (a) the order parameter versus the Monte Carlo reduced temperature $T^{*}$ and (b) the order parameter susceptibility versus $T^{*}$. The experimental curves presented in this figure assume the renormalization of the experimental random field $H_{\max }$ shown in Fig. 11.

whole study which brings new parameters, like the ones describing the moment distribution, the random field frequency compared with the Monte Carlo unit step, and even the choice of the numerical method itself (Monte Carlo might be not efficient since no temperature is applied). Another possible approach is to compute the average kinetic energy from the velocities measurements which would be of great interest and may be used to bear out the nonlinear evolution shown in Fig. 11.

\section{ACKNOWLEDGMENTS}

The authors would like to thank R. Ballou, G. Bertotti, J. L. Porteseil, and L. Thomas for helpful discussions. 
${ }^{1}$ L. Thomas, F. Lionti, R. Ballou, D. Gatteschi, R. Sessoli, and B. Barbara, Nature (London) 383, 145 (1996).

${ }^{2}$ J. R. Friedman, M. P. Sarachik, J. Tejada, and R. Ziolo, Phys. Rev. Lett. 76, 3830 (1996).

${ }^{3}$ C. Sangregorio, T. Ohm, C. Paulsen, R. Sessoli, and D. Gatteschi, Phys. Rev. Lett. 78, 4645 (1997).

${ }^{4}$ T. Ohm, C. Sangregorio, and C. Paulsen (unpublished).

${ }^{5}$ N. V. Prokof'ev and P. C. E. Stamp, Phys. Rev. Lett. 80, 5794 (1998).

${ }^{6}$ V. L. Pokrovsky, Adv. Phys. 28, 595 (1979).

${ }^{7}$ Y. M. Malozovsky and V. M. Rozenbaum, Physica A 175, 127 (1991).

${ }^{8}$ S. Romano, Phys. Scr. 50, 326 (1994).

${ }^{9}$ S. Prakash and C. L. Henley, Phys. Rev. B 42, 6574 (1990).

${ }^{10}$ J. Villain, R. Bidaux, J. P. Carton, and R. Conte, J. Phys. (Paris) 41, 1263 (1980).

${ }^{11}$ Lattices of compass needles (smaller ones) were first studied by $\mathrm{J}$. A. Ewing, [Philos. Mag. 30, 205 (1890)]. He was mainly interested in hysteresis cycles generated by these model systems of magnetism.

${ }^{12}$ P. I. Belobrov, R. S. Gekht, and V. A. Ignatchenko, Sov. Phys. JETP 57, 636 (1983).

${ }^{13}$ J. G. Brankov and D. M. Danchev, Physica A 144, 128 (1987).
${ }^{14}$ T. Erber and H. G. Latal, Bull. Cl. Sci., Acad. R. Belg. 53, 1019 (1967).

${ }^{15}$ V. E. Klymenko, V. M. Rozenbaum, V. V. Kukhtin, and O. V. Shramko, Solid State Commun. 88, 373 (1993).

${ }^{16}$ M. V. Feigel'man and A. M. Tsvelik, Sov. Phys. JETP 56, 823 (1982).

${ }^{17}$ R. Kretschmer and K. Binder, Z. Phys. B 34, 375 (1979).

${ }^{18}$ S. Romano, Nuovo Cimento D 9, 409 (1987).

${ }^{19}$ K. Binder, in Phase Transitions and Critical Phenomena, edited by C. Domb and M. S. Green (Academic Press, London, 1976), Vol. 5B.

${ }^{20}$ S. J. Knak Jensen and O. G. Mouritsen, Phys. Rev. Lett. 43, 1736 (1979).

${ }^{21}$ E. B. Priestley, P. J. Wojtowicz, and P. Sheng, in Introduction to Liquid Crystals, edited by E. B. Priestley, P. J. Wojtowicz and P. Sheng (Plenum Press, New York, 1974).

${ }^{22}$ C. Zannoni, in The Molecular Physics of Liquid Crystals, edited by G. R. Luckhurst and G. W. Gray (Academic Press, London, 1979), Chap. 3.

${ }^{23}$ D. P. Landau, Phys. Rev. B 13, 2997 (1976).

${ }^{24}$ M. E. Fisher and M. N. Barber, Phys. Rev. Lett. 28, 1516 (1972).

${ }^{25}$ K. Binder and P. C. Hohenberg, Phys. Rev. B 6, 3461 (1972). 